Parasitology

\title{
Rapid detection and simultaneous molecular profile characterization of Acanthamoeba infections ${ }^{2}$, 论访
}

\author{
Pablo Goldschmidt a,*, Sandrine Degorge ${ }^{a}$, Djida Benallaoua ${ }^{a}$, Laurence Batellier ${ }^{a}$, \\ David Di Cave ${ }^{\mathrm{b}}$, Christine Chaumeil ${ }^{\text {a }}$ \\ a Laboratoire du Centre Hospitalier National d'Ophtalmologie des Quinze-Vingts 28, rue de Charenton 75012 Paris, France \\ ${ }^{b}$ Department of Public Health and Cell Biology, University of Rome Tor Vergata, Rome, Italy
}

\section{A R T I C L E I N F O}

\section{Article history:}

Received 19 March 2012

Accepted 5 June 2012

Available online 20 July 2012

\section{Keywords:}

Acanthamoeba

Detection

High-resolution melting real-time PCR

Keratitis

Genotype

\begin{abstract}
A B S T R A C T
Diagnosis of Acanthamoeba by microscopic examination, culture, and polymerase chain reactions (PCRs) has several limitations (sensitivity, specificity, lack of detection of several strains, cost of testing for discrimination among strains). We developed a new high-resolution melting real-time PCR (HRM) to detect and characterize Acanthamoeba infections. HRM performances were evaluated with strains from the American Type Culture Collection (ATCC) and with 20 corneal scrapings. The DNA extracted from specimens were amplified, detected, and characterized in 1 run using 2 original primers diluted in a solution containing an intercalating dye. Detection and molecular characterization of Acanthamoeba infections could be achieved in less than $2.5 \mathrm{~h}$ with a dramatic reduction in cost of reactants (postamplification procedures and radioactive or fluorescentlabeled molecular probes were unnecessary). HRM detection limits were $0.1 \mathrm{cyst} / \mu \mathrm{L}$ or less (including genotypes T5 and T11), and its sensitivity and specificity were higher than other molecular tests. For the tested strains from the ATCC, the HRM drafted 4 different profiles: Type I (genotypes T2 and T4), Type II (T5 and T7), Type III (T8), and Type IV (T1, T3, T6, T9, T11, T12, and T13).
\end{abstract}

(c) 2012 Elsevier Inc. All rights reserved.

\section{Introduction}

Acanthamoebae are protozoa found in the soil, aquatic environment, and air. They are generally associated with eye diseases, although general infections (mostly brain and skin) were reported also. The infestations with Acanthamoeba are related to poor hygiene, inhalation of contaminated water, immunosuppression, corneal lens use, and corneal injuries (Lorenzo-Morales et al., 2010; Marciano Cabral, 2003).

To reduce morbidity, sensitive, specific, and inexpensive tests are necessary to administer therapeutic agents at the onset of symptoms. However, the direct smear analysis of corneal scrapings (Gram, MayGrünwald-Giemsa pH 7.4 staining) produces results rapidly, but misdiagnoses almost 60\% of clinical cases (Behets et al., 2006; Yera et al., 2007).

The detection of Acanthamoebae by polymerase chain reactions (PCR) dramatically enhanced diagnosis performances. The tests

\footnotetext{
Conflict of interests: none for all authors.

数论 Funding sources: This work was supported exclusively by intramural funds of the Laboratoire d'Ophtalmobiologie du Centre Hospitalier National d'Ophtalmologie des Quinze-Vingts, Paris, France.

* Corresponding author. Tel.: +33-1-40-02-16-94; fax: +33-1-40-02-16-99.

E-mail addresses: pablogol@aol.com, goldschmidt@quinze-vingts.fr (P. Goldschmidt).
}

targeting the Acanthamoeba nuclear small subunit ribosomal $18 \mathrm{~S}$ rRNA gene detected the equivalent of 1 protozoa per sample (Clarke and Niederkorn, 2006; Gatti et al., 2010; Marciano Cabral, 2003), but confirmation of the amplicon specificities required a series of additional procedures (agarose gel electrophoresis, hybridisation, and/or molecular sequencing), for which the opening of tubes with amplified material enhanced cross-contamination risks (Boggild et al., 2009; Goldschmidt et al., 2009a; Yera et al., 2007).

The reduction in postamplification handling was possible using a real-time technology (real-time PCR [rtPCR]), for which the amplicons produced during the rtPCR could be detected in closed tubes by means of 2 different strategies: a) fluorophore-labeled TaqMan probes or b) the intercalating fluorophore SYBR-green. Nevertheless, the SYBRgreen rtPCR did not fulfill the expectations for diagnosis because traces of residual double-stranded DNA from other agents found in the DNA extracted from the samples may bind the fluorophore and trigger nonspecific positive signals. Here, detecting fluorescent signals required the addition of postamplification procedures (agarose gel electrophoresis or sequencing) (Behets et al., 2006; Boggild et al., 2009) to determine species or genotypes. The use of labeled TaqMan ${ }^{\circledR}$ probes (Qvarnstrom et al., 2006; Yera et al., 2007) instead of SYBRgreen improved rtPCR specificity but did not detect all the genotypes. In addition, TaqMan rtPCRs were unable to provide group or genotype data (Da Rocha-Azevedo et al., 2009; Qvarnstrom et al., 2006). We reported a broad-spectrum rtPCR performed directly from clinical or 
environmental samples (targeting a highly conserved mitochondrial gene) that made possible the detection of all the available American Type Culture Collection (ATCC) strains ( 0.1 cyst/ $\mu \mathrm{L}$ of sample). Like other TaqMan rtPCRs, the broad-spectrum test that required expensive fluorophore-labeled probes was not informative on groups, species, types, or genotypes (Goldschmidt et al., 2009b).

The high-resolution melting real-time PCR technology (HRM) was developed for the fine assessment of DNA sequence variations (Monis et al., 2005; Reed et al., 2007) using improved fluorescent DNA-binding dyes. In this study, we developed a new molecular strategy based on HRM that should a) detect in 1 run the equivalent of 1 cyst of Acanthamoeba or less per sample; b) produce results without molecular probes based on the dye-melting derivative curve analysis with no need for gel electrophoresis, hybridizations, or immunoenzymatic assays; and c) draft different profiles for different species according to the guanine cytosine content and to the size of the amplicons neosynthesised during the nucleic-acid amplification process

\section{Material and methods}

HRM sensitivity, specificity, linearity, and detection limits were first assessed with strains of Acanthamoeba purchased from LGC Promochem (Molsheim, France; an ATCC partner) (A. comandoni [ref. 30135], A. palestinensis [ref. 50708], A. sp. [ref. 50655], A. palestinensis [ref. 30870], A. griffini [ref. 30731], A. castellanii [ref. 50373], A. lenticulata [ref. 30841], A. astronyxis [ref. 30137], A. tubiashi [ref. 30867], and $A$. hatchetti). Acanthamoeba were cultured and maintained according to the procedures indicated by the ATCC for each strain (Da Rocha-Azevedo et al., 2009; Marciano Cabral, 2003).

Investigations were conducted according to the principles expressed in the Declaration of Helsinki (http://www.wma.net/en/ 30publications/10policies/b3/index.html) and followed the requirements for approval by the Institutional Review Board of the Centre Hospitalier National des Quinze-Vingts (CHNO), Ministry of Public Health, Paris, France. For each sample, forms with written consent drafted according to the requirements of the CHNO Review Board and the National Health Authorities were sent to the laboratory after being double checked, validated, and signed by the physician in charge of the sampling.

After rinsing fluorescein and topical anesthetics from the eye surface, ophthalmologists performed deep scraping of the cornea with sterile stainless steel blades in order to obtain materials from patients presenting with corneal ulcers and requiring microbiological diagnosis (Goldschmidt et al., 2006, 2008). Samples were divided into aliquots and tested masked for the following:

a) Direct microscopic examination (specimens were fixed and stained with Giemsa [pH 7.4] and confirmed by Grocott's methenamine silver reaction);

b) Culture for aerobic and anaerobic bacteria and fungi. Isolates were phenotypically characterized by conventional tests (Chromagar Candida BR [ref. 257480], Becton Dickinson, Rungis, France; API 20AUX [ref. 20210], bioMérieux, Craponne, France; API Candida, bioMérieux; and Lactophenol Blue [ref. 363060-0125], RAL Advanced Chromatic, Martillac, France). Aliquots of each scraping were discharged into flasks with $5 \mathrm{~mL}$ of $0.9 \%$ sodium chloride and Escherichia coli suspensions and incubated up to 30 days before being discarded as culture negative (Da Rocha-Azevedo et al., 2009; Marciano Cabral, 2003; Yera et al., 2007):

c) DNA extraction for diagnosis of Herpesviridae by real-time PCR (Goldschmidt et al., 2006; Van Doornum et al., 2003) and of Acanthamoeba (Behets et al., 2006; Goldschmidt et al., 2008).

DNA was extracted in a vertical safety laminar flow cabinet. To monitor the yields of extraction and the PCR inhibitors, the internal control (IC) consisting of a virus preparation of seal herpes virus (Van
Doornum, Department of Virology Erasmus MC, Rotterdam, The Netherlands) was added before extraction and $200 \mu \mathrm{L}$ was used for the extraction on a MagNA Pure compact instrument (Roche, Meylan, France) (Goldschmidt et al., 2008; Van Doornum et al., 2003). DNA extracts were tested by 2 rtPCRs targeting different genes (Behets et al., 2006; Goldschmidt et al., 2009a, b; Qvarnstrom et al., 2006) and by HRM. The primers selected for HRM bracket significant polymorphisms in the mitochondrion gene (gi|562028|gb|U12386.1| ACU12386, U12386, U12386.1GI: 562028). Forward and reverse HRM primer sequences are 5'GCAGTCGCGGTAATACGA and 5' ACCACCTACGCACCCTTTACA, respectively.

Kits prepared for HRM consist of 2 tubes: the first for detection, semiquantification, and molecular profile characterization of Acanthamoeba (10 $\mu \mathrm{L}$ of MeltDoctor ${ }^{\circledR}$ HRM Master Mix [ABI ref. 4415440, Applied Biosystems, France] and $1 \mu \mathrm{L}$ each of the forward and the reverse primers, each at $300 \mathrm{nmol} / \mathrm{L}$ final concentration); and the second for amplification of the IC (seal herpes). HRM was performed by introducing $10 \mu \mathrm{L}$ of the DNA extract in the tube for Acanthamoeba detection and $10 \mu \mathrm{L}$ in the tube for the IC.

HRM amplicon production and size were automatically assessed with the $A B I 7500$ upgraded equipment. The amplification program consisted in one cycle of $10 \mathrm{~min}$ at $95^{\circ} \mathrm{C}$, followed by 55 cycles $(15 \mathrm{~s}$ at $95^{\circ} \mathrm{C}, 30 \mathrm{~s}$ at $60^{\circ} \mathrm{C}$, and $30 \mathrm{~s}$ at $72^{\circ} \mathrm{C}$ ). The HRM dye melting curve was obtained by heat denaturation $\left(95^{\circ} \mathrm{C}\right.$ for $15 \mathrm{~s}$, cooling to $50^{\circ} \mathrm{C}$ for $1 \mathrm{~min}$, and a temperature increase until $60^{\circ} \mathrm{C}$ for $15 \mathrm{~s}$ with a $2.2^{\circ} \mathrm{C} / \mathrm{s}$ ramp rate). The difference plot was used for amplicon analysis, and the specificity of the assay was guaranteed by the right peak and the additional melting analysis. The first derivative of the melting curves and the "melt peaks" (Tm) allowed establishing comparisons between clinical isolate profiles and the referenced strains from the ATCC. Samples with fluorescence of less than $100 \%$ of the maximum were excluded from the analysis. The internal IC was amplified in an independent real-time PCR run (Goldschmidt et al., 2008, 2009b; Van Doornum et al., 2003).

HRM specificity was assessed with DNA extracts obtained by mixing $100 \mu \mathrm{L}$ of suspensions of $10^{6} / \mathrm{mL}$ of human fibroblasts or $10^{6}$ $\mathrm{CFU} / \mathrm{mL}$ of different bacteria or fungi or $10^{6} \mathrm{PFU} / \mathrm{mL}$ of herpes simplex virus (HSV) type 1 and type 2, or varicella zoster virus (VZV) or Adenovirus type 8 with or without 20 Acanthamoeba cysts.

Genotyping of Acanthamoeba was carried out by amplification and analysis of the ASA.S1 region of the 18S rRNA gene using the genusspecific primers JDP1 and JDP2 (Goldschmidt et al., 2009b; Marciano Cabral, 2003). Sequences were aligned with a standard set of sequences, and the analysis of the nearly complete Rns genes allowed genotype classification. Bacteria, fungi, or viruses were characterized with kits validated for clinical diagnosis. Clinical samples were read masked by 2 trained technicians and confirmed by 2 members of the medical staff of the clinical laboratory.

\section{Results}

The selected primers detected Acanthamoeba with a linear range spanning from $10^{5}$ to at least $10^{-1}$ cyst $/ \mathrm{mL}$. The interassay reproducibility coefficient variation was less than 10\% among HRM runs for each dilution. The HRM coefficient of variation for the interassay reproducibility in the complete linear range of detection ( $10^{5}$ to $1 \mathrm{cyst} / \mathrm{mL}$ ) for 5 runs was inferior to $10 \%$.

The reactants, disposable material, and environmental controls did not produce signals that could have interfered with the interpretation of results. Human cells and several human pathogens did not interfere with HRM (Staphylococcus epidermidis and aureus, Streptococcus pneumoniae, Serratia marcescens, E. coli, Pseudomonas acnes, P. aeruginosa, Candida albicans, Fusarium sp., Aspergillus sp., HSV-1 and -2, VZV, and Adenovirus type 8).

As shown in Table 1, HRM detected all the strains (detection limits: $10^{-1}$ cysts/ $\mu \mathrm{L}$ or less) and drafted simultaneously 4 different consistent profiles: Profile I for genotypes T2 and T4; Profile II for T5 
Table 1

Comparison of Acanthamoeba nuclear 18S ribosomal RNA genotypes with highresolution melting analysis (HRM).

\begin{tabular}{llll}
\hline Strain & Genotype & Result (1 cyst/reaction) & HRM \\
\hline A.castellani & T4 & Positive & Profile 1 \\
A. palestinensis & T2 & Positive & Profile 1 \\
A. palestinensis 50708 & T2 & Positive & Profile 1 \\
A. astronyxis & T7 & Positive & Profile 2 \\
A. lenticulata & T5 & Positive & Profile 2 \\
A. tubiashi & T8 & Positive & Profile 3 \\
A. comandoni & T9 & Positive & Profile 4 \\
A. griffini & T3 & Positive & Profile 4 \\
A. hatchetti & T11 & Positive & Profile 4 \\
A. sp* & T1 & Positive & Profile 4 \\
A. sp* & T6 & Positive & Profile 4 \\
A. sp* & T13 & Positive & Profile 4 \\
A. sp* & T14 & Positive & Profile 4 \\
A. healyi & T12 & Positive & Profile 4 \\
\hline
\end{tabular}

* Isolates from Acanthamoeba keratitis tested masked (5 different experiments for each).

and T7; Profile III for T8; and Profile IV for genotypes T1, T3, T6, T9, T11, T12, T13, and T14 (Table 1). Compared to controls, the Ct values for the IC were never delayed for more than 1.5 cycles.

In samples obtained from patients with Acanthamoeba keratitis, the direct microscopic examination detected cysts in 50\% and 60\% which were Acanthamoeba culture positive. The time for culture positive ranged between 2 and 4 weeks.

For each sample, the results were validated only if the $\mathrm{Ct}$ value obtained for the IC compared to the blank was not delayed by more than 1.5 cycles. The $\mathrm{Ct}$ values obtained here for the ICs indicate that the yields of extraction of DNA were satisfactory and that the potential PCR inhibitors were eliminated. As shown in Table 2, HRM was negative for all the samples testing negative for Acanthamoeba culture, negative for Acanthamoeba TaqMan rtPCRs targeting the ribosomal (Qvarnstrom et al., 2006; Rivière et al., 2006) or the mitochondrial gene (Goldschmidt et al., 2009a), and negative for the SYBR-green rtPCRs.

The 2 SYBR-green rtPCRs were positive for all the samples from Acanthamoeba keratitis (samples 1 to 10 ), but the signal analysis did not allow establishing molecular profiles. SYBR-Green specificity was $85 \%$. Sensitivity and specificity for the TaqMan rtPCR targeting the ribosome (Qvarnstrom et al., 2006) were 90\% for both (negative results for genotype T5 and very weak signals for genotypes T1 and T3). The ribosome-targeted rtPCR repeatedly produced falsepositive results for samples containing S. lexinton, Clostridium sporulans, and P. acnes. Sensitivity and specificity for the TaqMan rtPCR targeting the mitochondrion (Goldschmidt et al., 2009a, b) were both $100 \%$ (Table 2 ).

Table 3 presents the HRM profiles compared to ribosome gene sequencing. Clinical isolates here were largely (70\%) T4 and T3 as previously reported for AK (Booton et al., 2005; Lorenzo-Morales et al., 2010; Marciano-Cabral, 2009) and 10\% were genotype T5 and $10 \%$ were T11. Eight of 10 HRM profiles correspond to what was expected from ribosome sequencing, but 2 isolates characterized as genotype T4 showed unexpected type I HRM profile.

\section{Discussion}

Mixing the DNA extracted from samples with a solution containing primers from a selected sequence and 1 intercalating dye that stoichiometric binds reversibly to the neoformed double-stranded DNA during amplification makes it possible to conduct an automatic melting analysis to detect Acanthamoeba that provides 4 reproducible signatures for the different genotypes. The detection of Acanthamoeba and their molecular characterization are performed by obviating gel electrophoresis, hybridizations, immunoassays, etc., because the automatic melting analysis amplified with 1 set of primers suited consistently to discriminate among 4 Acanthamoeba mitochondrial

Table 2

Direct microscopic examination, culture, real-time PCR, and HRM performances on samples obtained from patients with keratitis.

\begin{tabular}{|c|c|c|c|c|c|c|c|}
\hline \multirow[t]{3}{*}{ No. } & \multicolumn{7}{|l|}{ Test } \\
\hline & $\begin{array}{l}\text { Direct microscopic examination } \\
\text { of stained scrapings }\end{array}$ & $\begin{array}{l}\text { Culture } \\
\text { (time for positivity, weeks) }\end{array}$ & $\begin{array}{l}\text { TaqMan } \\
\text { real-time } \mathrm{PCR}^{\mathrm{a}, \mathrm{c}}\end{array}$ & $\begin{array}{l}\text { TaqMan } \\
\text { real-time } \mathrm{PCR}^{\mathrm{a}, \mathrm{d}}\end{array}$ & $\begin{array}{l}\text { SYBR Green } \\
\text { real-time PCR }\end{array}$ & HRM & Genotype+ \\
\hline & \multicolumn{7}{|l|}{ Results } \\
\hline 1 & Positive & Positive, 2 & Positive & Positive** & Positive & Positive & $\mathrm{T} 4$ \\
\hline 2 & Positive & Negative & Positive \#\# & Positive** & Positive & Positive & $\mathrm{T} 11$ \\
\hline 3 & Negative & Negative & Positive \#\# & Positive** & Positive & Positive & T3 \\
\hline 4 & Negative & Positive, 3 & Negative & Positive** & Positive & Positive & $\mathrm{T} 5$ \\
\hline 5 & Positive & Positive, 3 & Positive & Positive** & Positive & Positive & $\mathrm{T} 4$ \\
\hline 6 & Positive & Negative & Positive & Positive** & Positive & Positive & $\mathrm{T} 4$ \\
\hline 7 & Negative & Positive, 4 & Positive & Positive** & Positive & Positive & $\mathrm{T} 4$ \\
\hline 8 & Positive & Positive, 2 & Positive & Positive** & Positive & Positive & $\mathrm{T} 4$ \\
\hline 9 & Negative & Negative & Positive & Positive** & Positive & Positive & $\mathrm{T} 4$ \\
\hline 10 & Negative & Positive;3 & Positive & Positive** & Positive & Positive & $\mathrm{T} 4$ \\
\hline 11 & Negative & P. aeruginosa & Negative & Negative & Negative & Negative & \\
\hline 12 & Negative & S. aureus & Negative & Negative & Negative & Negative & \\
\hline 13 & Negative & S. lexinton & False positive \#\# & Negative & False positive \#\# & Negative & \\
\hline 14 & Negative & S. pyogenes & Negative & Negative & Negative & Negative & \\
\hline 15 & Negative & P. aeruginosa & Negative & Negative & Negative & Negative & \\
\hline 16 & Negative & A. freundii & Negative & Negative & Negative & Negative & \\
\hline 17 & Negative & C. sporulans & Negative & Negative & False positive \#\# & Negative & \\
\hline 18 & Negative & S. marcescens & Negative & Negative & Negative & Negative & \\
\hline 19 & Negative & P. acnes $+S$. lexinton & False positive \#\# & Negative & False positive \#\# & Negative & \\
\hline 20 & Negative & E. coli & Negative & Negative & Negative & Negative & \\
\hline
\end{tabular}

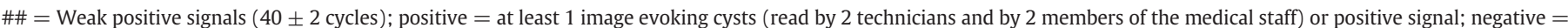
negative signal; ${ }^{* *}=\mathrm{Ct}<35 ;+=$ nuclear $18 \mathrm{~S}$ ribosomal RNA gene sequencing.

a Internal control $\mathrm{Ct}$ values ranged between 31.3 and 33.1.

b Peak at $78^{\circ} \mathrm{C}$.

c Qvarnstrom et al. (2006).

d Goldschmidt et al. (2009a). 
Table 3

Genotyping analysis of corneal isolates (ribosomal sequences) ${ }^{\mathrm{a}}$ compared to HRM. $^{\mathrm{b}}$

\begin{tabular}{lll}
\hline Clinical isolate genotype $^{\mathrm{a}}$ & HRM profile $^{\mathrm{b}}$ & $\begin{array}{l}\text { Expected profile } \\
\text { according to sequencing }\end{array}$ \\
\hline T4 & 4 & $1^{\mathrm{c}}$ \\
T11 & 4 & 4 \\
T3 & 4 & 4 \\
T5 & 2 & 2 \\
T4 & 1 & 1 \\
T4 & 4 & $1^{\mathrm{c}}$ \\
T3 & 4 & 4 \\
T4 & 1 & 1 \\
T4 & 1 & 1 \\
T4 & 1 & 1 \\
\hline
\end{tabular}

a The amplification of the ASA.S1 region of the 18S rRNA gene using the primers JDP1 and JDP2 was followed by sequence reactions, capillary electrophoresis, and molecular alignments (Booton et al., 2009, Goldschmidt et al., 2009b).

b HRM molecular profiles were directly obtained from the corneal samples.

Discordances between HRM mitochondrial sequence analysis and ribosomal molecular sequencing.

gene groups. The classic PCRs and the TaqMan rtPCRs are unable to detect all the Acanthamoeba species (Boggild et al., 2009; Goldschmidt et al., 2009a; Ledee et al., 2003; Qvarnstrom et al., 2006), and, therefore, additional procedures should be carried out to avoid false conclusions. On the other hand, SYBR rtPCRs have a limited predictive value because false-positive signals are repeatedly obtained with residual nucleic acids present in the DNA extracts and false-negative results were reported (Taq-DNA polymerase inhibition by the fluorophore) (Boggild et al., 2009; Monis et al., 2005; Reed et al., 2007).

The new HRM a) detects at least $0.1 \mathrm{cyst} / \mathrm{mL}$ of all the tested strains of Acanthamoeba; b) confirms diagnosis directly from clinical samples; c) assesses simultaneously the yields of DNA extraction and the PCR inhibitors; d) drafts 4 different molecular profiles; e) is performed without molecular probes (radioactive or nonradioactive); and f) allows results with molecular characterizations to be obtained in less than $2.5 \mathrm{~h}$ after DNA extraction; $\mathrm{f}$ ) is not modified by high loads of bacteria, fungi, herpes simplex viruses, adenoviruses, or human cells. HRM allowed corneal samples to be readily assayed after DNA extraction without interference from DNA sequences that may be found in the specimen. For the series of 20 corneal scrapings tested here, HRM detecting capacities and specificity were higher than culture and all other nucleic-acid amplification techniques (Behets et al., 2006; Da Rocha-Azevedo et al., 2009; Rivière et al., 2006). The loop-mediated isothermal amplification (LAMP) is a method that does not require thermocyclers and DNA purification procedures. LAMP was recently developed for the diagnosis of Trypanosoma spp., Cryptosporidium spp., Theileria, canine and equine piroplasmosis, Toxoplasma gondii, Giardia duodenalis, Microsporidia, Taenia sp., and Plasmodia (Karanis and Ongerth, 2009) (Baldursson and Karanis, 2011). Further investigations should be conducted to compare HRM and LAMP performances for the detection, quantification, and characterization of Acanthamoeba infections.

Major limitations were reported for epidemiologic and physiopathology studies, for which the taxonomy of Acanthamoeba was based on cyst morphology and trophozoite size and shape. In fact, protozoa morphology changes according to culture conditions and, therefore, further phylogenetic analysis was conducted according to the nuclear $18 \mathrm{~S}$ ribosomal RNA gene (Rns or 18S rDNA) sequence that considered that a $5 \%$ dissimilarity value separates genotypes (Goldschmidt et al., 2009b; Ledee et al., 2003; Marciano Cabral, 2003). The Rns trees compared with those obtained by sequencing the mitochondrial small subunit rRNA gene (Rns or 16S rDNA) failed to distinguish between genotypes T3 and T11. HRM (without electrophoresis, hybridization, sequencing reactions, or any additional laborious postamplification manipulation) drafted similar profiles (HRM profile type IV) for samples containing the Rns genotypes T3 and T11.

The ribosomal 18S rDNA Rns phylogenetic relationships identified a major clade designated $\mathrm{T} 4$, which included 53 different strains having 22 different sequences (Marciano-Cabral, 2009; Booton et al., 2009). In the present study, 2 clinical samples characterized by Rns sequencing as T4 produced an unexpected HRM type IV profile (instead of type I). These results may confirm previous reports suggesting that strains from the T4 clade could be distinguished with a higher level of significance for trees built according to the Rns mitochondria-associated sequences (Ledee et al., 2003). In the present study, the genotype T4 was detected in $70 \%$ of keratitis, from which only $50 \%$ produced the expected $\mathrm{T} 4$ HRM profile. HRM may therefore provide relevant additional information for the comprehensive classification of protozoa (Ledee et al., 2003; Marciano Cabral, 2003) and confirm hypothesis suggesting that the named taxa based on $18 \mathrm{~S}$ rDNA Rns phylogenetic relationship genotyping are not monophyletic entities. In previous $18 \mathrm{~S}$ rDNA-based reports, the genotype T4 was predominant ( $85 \%)$ (Boggild et al., 2009; Booton et al., 2009; Ledee et al., 2003; Lorenzo-Morales et al., 2010; Schroeder et al., 2001; Zhao et al., 2010). However, in Greece, the genotypes T2, T3, T5, T6, and T11 have been identified (Spanakos et al., 2006) and in Slovakia and the Czech Republic the genotypes T15, T4, and T3 were detected in AK (Nagyová et al., 2010). In Italy, 25\% of the global Acanthamoeba keratitis isolates were identified as T15 (Di Cave et al., 2009).

The Acanthamoeba cultures require time, rely on the ability of the protozoa to grow ex vivo, are time consuming, and may become positive late in the course of the infection. Great improvement was obtained using TaqMan rtPCR tests but they are unable to detect strains from genotype T5 (Qvarnstrom et al., 2006) and A. astronyxis (Da Rocha-Azevedo et al., 2009; Qvarnstrom et al., 2006). Moreover, the cost for the numerous TaqMan probes and for the postamplification procedures required for characterizing Acanthamoeba is high (Gatti et al., 2010; Goldschmidt et al., 2009b; Khairnar et al., 2011; Rivera and Adao, 2009; Schroeder et al., 2001; Yera et al., 2007).

As shown in Table 3, HRM profiles I and IV have the potential to cause keratitis (samples from patients presenting with Acanthamoeba keratitis). However, the sample size of this study is too small to state general conclusions, and, therefore, further investigations in different settings with a larger series of samples are necessary to confirm the association of each of the 4 HRM profiles with pathology, tropism, and environmental distribution.

In conclusion, HRM detects Acanthamoeba (including genotypes T5 and T11) and drafts molecular profiles with higher sensitivity than culture and other molecular tools. It has the relevant advantage of minimizing cross-contamination risks because the amplification procedures and the signal detection and the DNA melting analyses are carried out in closed tubes. In addition, HRM minimizes the risks for false-negative conclusions because the yields of extraction of DNA and the PCR inhibitors are systematically monitored. The cost for reactants for testing 1 DNA extract (detection and molecular characterization of Acanthamoeba) could be reduced to less than US $\$ 2.00$ because HRM can be carried out with the equipment routinely used in laboratories for real-time PCR (upgrading the software required) with no need for molecular probes, gels, restriction enzymes, or any additional equipment for sophisticated postamplification procedures. Future analyses should generate reproducible melt curves over time in different settings to build reference databases to store HRM calculations and shapes of the melting profiles for each family or species in order to be challenged against the results of future testing. On the above, larger trials are necessary to confirm the usefulness of HRM for environmental studies and disease management (Baldursson and Karanis, 2011; Karanis and Ongerth, 2009). 


\section{Acknowledgments}

The authors thank the Centre de Ressources Biologiques du Centre Hospitalier National d'Ophtalmologie des Quinze-Vingts, Paris, France, for providing the biological samples.

\section{References}

Baldursson S, Karanis P. Waterborne transmission of protozoan parasites: review of worldwide outbreaks-an update 2004-2010. Water Res 2011;15:6603-14.

Behets J, Declerck P, Delaedt Y, et al. Quantitative detection and differentiation of freeliving amoeba species using SYBR green-based real-time PCR melting curve analysis. Curr Microbiol 2006:53:506-9.

Boggild AK, Martin DS, Lee TY, et al. Laboratory diagnosis of amoebic keratitis: comparison of four diagnostic methods for different types of clinical specimens. J Clin Microbiol 2009;47:1314-8.

Booton GC, Visvesvara GS, Byers TJ, et al. Identification and distribution of Acanthamoeba species genotypes associated with nonkeratitis infections. J Clin Microbiol 2005;43:1689-93.

Booton G, Joslin C, Shoff M, et al. Genotypic identification of Acanthamoeba sp. isolates associated with an outbreak of acanthamoeba keratitis. Cornea 2009;28:673-6.

Clarke DW, Niederkorn JY. The pathophysiology of Acanthamoeba keratitis. Trends Parasitol 2006;22:175-80.

da Rocha-Azevedo B, Tanowitz HB, Marciano-Cabral F. Diagnosis of infections caused by pathogenic free-living amoebae. Interdiscip Perspect Infect Dis 2009;2009:251406.

Di Cave D, Monno R, Bottalico P, Guerriero, et al. Acanthamoeba T4 and T15 genotypes associated with keratitis infections in Italy. Eur J Clin Microbiol Infect Dis 2009;28: 607-12.

Gatti S, Rama P, Matuska S, et al. Isolation and genotyping of Acanthamoeba isolates from corneal infections in Italy. J Med Microbiol 2010;12:20705732.

Goldschmidt P, Rostane H, Saint-Jean C, et al. Effects of topical anaesthetics and fluorescein on the real-time PCR used for the diagnosis of herpesviruses and Acanthamoeba keratitis. Br J Ophthalmol 2006;90:1354-6.

Goldschmidt P, Degorge S, Saint-Jean C, et al. Resistance of Acanthamoeba to classic DNA extraction methods used for the diagnosis of corneal infections. Br J Ophthalmol 2008;92:112-5.

Goldschmidt P, Dardé M, Yera $\mathrm{H}$, et al. Rapid detection and characterization of pathogens by molecular techniques. In: Liu Dongyou, editor. Molecular detection of foodborne pathogens; Chapter 45: Acanthamoeba. CRC Press1420076434; 2009a.

Goldschmidt P, Degorge S, Benallaoua D, et al. New tool for the simultaneous detection of 10 different genotypes of Acanthamoeba available from the American Type Culture Collection. Br J Ophthalmol 2009b;93:1096-100.
Karanis P, Ongerth J. LAMP: a powerful and flexible tool for monitoring microbial pathogens. Trends Parasitol 2009:25:498-9.

Khairnar K, Tamber G, Ralevski F, Pillai D. Comparison of molecular diagnostic methods for the detection of Acanthamoeba spp. from clinical specimens submitted for keratitis. Diagn Microbiol Infect Dis 2011;70:499-506.

Ledee DR, Booton GC, Awwad MH, et al. Advantages of using mitochondrial 16S rDNA sequences to classify clinical isolates of Acanthamoeba. Invest Ophthalmol Vis Sci 2003;44:1142-9.

Lorenzo-Morales J, Marciano-Cabral F, Lindo JF, et al. Pathogenicity of amoebae. Exp Parasitol 2010;126:2-3.

Marciano Cabral F. Acanthamoeba spp. as agents of disease in humans. Clin Microbiol Rev 2003;16:273-307.

Marciano-Cabral F. Free-living amoebae as agents of human infection. J Infect Dis 2009;15(199):1104-6.

Monis P, Giglio S, Saint C. Comparison of SYT09 and SYBR Green I for real-time polymerase chain reaction and investigation of the effect of dye concentration on amplification and DNA melting curve analysis. Anal Biochem 2005;340:24-34.

Nagyová V, Nagy A, Timko J. Morphological, physiological and molecular biological characterisation of isolates from first cases of Acanthamoeba keratitis in Slovakia. Parasitol Res 2010;106:861-72.

Qvarnstrom Y, Visvesvara G, Sriram R, da Silva A. Multiplex real-time PCR assay for the simultaneous detection of of Acanthamoeba spp., Balamuthia mandrillaris, and Naegleria fowleri. J Clin Microbiol 2006;44:3589-95.

Reed G, Kent J, Wittwer C. High-resolution DNA melting analysis for simple and efficient molecular diagnostics. Pharmacogenomics 2007;8:597-608.

Rivera W, Adao D. 18S ribosomal DNA genotypes of Acanthamoeba species isolated from contact lens cases in the Philippines. Parasitol Res 2009;105:1119-24.

Rivière D, Szczebara F, Berjeaud J, et al. Development of a real-time PCR assay for quantification of Acanthamoeba trophozoites and cysts. J Microbiol Methods 2006;64:78-83.

Schroeder J, Booton G, Hay J, et al. Use of subgenic 18S ribosomal DNA PCR and sequencing for genus and genotype identification of Acanthamoeba from humans with keratitis and from sewage sludge. J Clin Microbiol 2001;39:1903-11.

Spanakos G, Tzanetou K, Miltsakakis D, et al. Genotyping of pathogenic Acanthamoebae isolated from clinical samples in Greece: report of a clinical isolate presenting T5 genotype. Parasitol Int 2006;55:147-9.

Van Doornum G, Guldemeester J, Osterhaus ADME, et al. Diagnosing herpesvirus infections by real-time amplification and rapid culture. J Clin Microbiol 2003;41: 2576-80.

Yera H, Zamfir O, Bourcier T, et al. Comparison of PCR, microscopic examination and culture for the early diagnosis and characterization of Acanthamoeba isolates from ocular infections. Eur J Clin Microbiol Infect Dis 2007;26:221-4

Zhao G, Sun S, Zhao J, Xie L. Genotyping of Acanthamoeba isolates and clinical characteristics of patients with Acanthamoeba keratitis in China. J Med Microbiol 2010;59:462-6. 